\title{
The influence of Debye plasma on the ground state energies of exotic systems
}

\author{
Amar N. Sil ${ }^{a, c}$, Mariusz Pawlak $^{\mathrm{b}}$, Prasanta K. Mukherjee ${ }^{\mathrm{a}, *}$, \\ and Mirosław Bylicki ${ }^{\mathrm{b}}$ \\ ${ }^{\mathrm{a}}$ Indian Association for the Cultivation of Science, \\ Jadavpur, Kolkata-700 032, India \\ ${ }^{\mathrm{b}}$ Instytut Fizyki, Uniwersytet Mikotaja Kopernika, \\ ul. Grudziadzka 5, PL-87-100 Toruń, Poland \\ ${ }^{\mathrm{c}}$ Max Planck Institute for the Physics of Complex Systems, \\ Noethnitzer Str. 38, D-01187 Dresden, Germany
}

\begin{abstract}
The effect of plasma environment on the ground state energies of exotic systems $p p \mu, d d \mu$ and $t t \mu$ has been analyzed within a generalized three-body formalism using multi-term correlated basis sets. The Debye screening model of the plasma has been adopted for such a study. The binding energies of $p$ with $p \mu, d$ with $d \mu$ and $t$ with $t \mu$ have been estimated for a range of values of the Debye screening parameters. The systems tend towards instability for increased screening. The effect of particle correlation has been investigated in detail and is found to play important role for the stability in these systems.
\end{abstract}

Key words: exotic systems, Debye plasma, three-body system, quantum confinement

PACS: 36.10.-k

\section{Introduction}

Study of the effect of external environment like that of a plasma on the energy levels and other structural properties of atomic, ionic and exotic systems has become a subject of extensive investigations in recent years [1-12]. A broad

* Corresponding author.

Email address: sppkm@iacs.res.in (Prasanta K. Mukherjee). 
discussion on the subject matter was given by Griem [13], Fujimoto [14], Ichimaru [15] and Rajagopal [16]. Depending on the density and temperature of the external plasma, one can adopt various models of plasma-atom interaction whose effect is to alter the potential energy compared to that of the free atom. For low density and high temperature one usually considers the Debye screening model [17] while for high density and low temperature the ion sphere model [18] is usually adopted.

In this work we are interested in studying the ground state energy of the Coulombic three-body muonic molecular ions $p p \mu, d d \mu$ and $t t \mu$ under an external plasma environment. These systems are particularly important in the muon catalyzed fusion processes [19-21]. Muonic molecular ions with protonic substitutions are less adiabatic than the corresponding hydrogen molecular ions due to the mass difference between muon and proton. Such systems are of general interest theoretically $[22,23]$. During the passage of particles through matter most of these exotic hadronic systems are formed, albeit, their low mean lives [20,24,25]. One can consider the background to mimic a plasma. Hence one can apply a plasma model for estimating the properties of the exotic systems in such an environment, particularly for a fusion plasma. Although a number of highly accurate theoretical estimates are available for the bound state properties of such free exotic three-body systems [26-29], calculations predicting the behavior in presence of plasma are still scanty [30-33].

In the current communication we use the methodology adopted earlier [32] for the estimation of the energy of the exotic systems $p p \mu, d d \mu$ and $t t \mu$ in their spherically symmetric ground states in presence of plasma. Coupling to the plasma is included by the Debye screening model [17] in which the potential of the interaction between the charged particles is represented by screened Coulomb potential. We use the Ritz variational method in which the trial wave function is a linear combination of product basis functions. The particle correlation is taken care of by introducing explicitly the interparticle coordinate into the basis functions. The behavior of the energy of the system is analyzed with respect to the Debye screening constant. We aim at predicting an overall but unambiguous behavior of the ground state energies by using reasonably good basis sets such that the computation time is reasonable.

\section{Method}

The system consisting of a muon and two heavier particles is displayed in Fig. 1. The particles are placed along the three corners of a triangle with the muon at the origin. Their masses are $m_{3}=m_{\mu}=206.7682657 m_{e}$ and $m_{1}=m_{2}=\left\{m_{p}, m_{d}\right.$ or $\left.m_{t}\right\}$ depending on the system under study with $m_{p}=1836.1526675 m_{e}, m_{d}=3670.4829550 m_{e}$ and $m_{t}=5496.92158 m_{e}$. We 
consider nonrelativistic Hamiltonian of such a system embedded in a Debye plasma. Thus the Coulomb interaction between particles is screened by plasma and reads

$$
V=-\frac{e^{-\lambda r_{1}}}{r_{1}}-\frac{e^{-\lambda r_{2}}}{r_{2}}+\frac{e^{-\lambda r_{12}}}{r_{12}}
$$

The Debye screening constant is represented by [17]

$$
\lambda=\left[\frac{4 \pi(1+Z) n}{\kappa T}\right]^{\frac{1}{2}},
$$

where $n$ is the density number, $T$ is the temperature of the plasma and $Z$ is the nuclear charge (which is unity in the present case). The screening parameter can be adjusted by using suitable values of plasma density and temperature.

For the spherically symmetric ground state of a three-body system, momentum conservation leads to a Hamiltonian expressible in terms of relative coordinates $r_{1}, r_{2}$ and $r_{12}$ and the expectation value of the Hamiltonian with respect to a real and normalized wavefunction $\Psi$ can be represented as

$$
\begin{aligned}
& \langle\Psi|H| \Psi\rangle=\int\left\{\frac{1}{2}\left(\frac{1}{m_{1}}+\frac{1}{m_{3}}\right)\left(\frac{\partial \Psi}{\partial r_{1}}\right)^{2}+\frac{1}{2}\left(\frac{1}{m_{2}}+\frac{1}{m_{3}}\right)\left(\frac{\partial \Psi}{\partial r_{2}}\right)^{2}\right. \\
& +\frac{1}{2}\left(\frac{1}{m_{1}}+\frac{1}{m_{2}}\right)\left(\frac{\partial \Psi}{\partial r_{12}}\right)^{2}+\frac{1}{m_{3}}\left(\frac{r_{1}^{2}+r_{2}^{2}-r_{12}^{2}}{2 r_{1} r_{2}}\right)\left(\frac{\partial \Psi}{\partial r_{1}}\right)\left(\frac{\partial \Psi}{\partial r_{2}}\right) \\
& +\frac{1}{m_{2}}\left(\frac{r_{2}^{2}-r_{1}^{2}+r_{12}^{2}}{2 r_{2} r_{12}}\right)\left(\frac{\partial \Psi}{\partial r_{2}}\right)\left(\frac{\partial \Psi}{\partial r_{12}}\right) \\
& +\frac{1}{m_{1}}\left(\frac{r_{1}^{2}-r_{2}^{2}+r_{12}^{2}}{2 r_{1} r_{12}}\right)\left(\frac{\partial \Psi}{\partial r_{1}}\right)\left(\frac{\partial \Psi}{\partial r_{12}}\right) \\
& \left.+\left(-\frac{e^{-\lambda r_{1}}}{r_{1}}-\frac{e^{-\lambda r_{2}}}{r_{2}}+\frac{e^{-\lambda r_{12}}}{r_{12}}\right) \Psi^{2}\right\} \\
& r_{1} r_{2} r_{12} d r_{1} d r_{2} d r_{12}
\end{aligned}
$$

To minimize the expectation value, the wavefunction $\Psi$ is expanded in terms of the interparticle coordinates which takes care of the electron correlation effect explicitly

$$
\Psi\left(r_{1}, r_{2}, r_{12}\right)=\sum_{k l j} C_{k l j} \chi_{k l}(1,2) \eta_{j}(1,2)
$$


where

$$
\chi_{k l}(1,2) \sim\left(e^{-\rho_{k} r_{1}-\rho_{l} r_{2}}+e^{-\rho_{l} r_{1}-\rho_{k} r_{2}}\right)
$$

constructed of Slater type orbitals are to take care of the radial correlation whereas

$$
\eta_{j}(1,2) \sim r_{12}^{n_{j}} e^{-\beta_{j} r_{12}}
$$

describe the angular proton-proton $(d-d$ or $t-t)$ correlations dependent on the distance between them, $r_{12}$. In actual computations we used eight different exponent parameters $\rho$ which resulted in 36 different $\chi_{k l}$ functions. For the angular expansion we used nine different $\eta$ 's leading to altogether $36 \times 9=324$ linear variation coefficients for the fully correlated calculations. The linear expansion, Eq. (3), leads to the matrix generalized eigenvalue problem

$$
\underline{\underline{\mathbf{H}}} \underline{\mathbf{C}}=E \underline{\underline{S}} \underline{\mathbf{C}},
$$

where $\underline{\underline{\mathbf{H}}}$ and $\underline{\underline{\mathbf{S}}}$ are the Hamiltonian and overlap matrices built in terms of basis functions $\chi_{k l} \eta_{j}$. In general, the nonlinear parameters $\rho$ and $\beta$ can be suitably adjusted so as to minimize the ground state eigenvalue from Eq. (4). We have optimized them for the plasma-free case.

\section{Results}

We have considered here the plasma confined exotic systems $p p \mu, d d \mu$ and $t t \mu$ bound by Coulomb interactions. The ground state energies computed as described above are given in Tables 1, 2 and 3, respectively for $p p \mu, d d \mu$ and $t t \mu$. The energies of the plasma free systems $(\lambda=0)$ can be compared with very accurate results due to Frolov [27]. Reasonable agreement is observed: seven, six and five figures respectively for $p p \mu, d d \mu$ and $t t \mu$.

To decide whether the system is bound or not one needs to compare the three-particle energy to the ground state level of a corresponding two-particle system $(p \mu, d \mu$, and $t \mu$ respectively for $p p \mu, d d \mu$ and $t t \mu)$. We computed the ground state energies of the two-body systems variationally in a basis of eight Slater type orbitals (which is adequate to the basis described above, used for three-body computations). They also are given in Tables $1-3$. The results for both two- and three-particle systems containing protons are plotted in Fig. 2. For two other cases $(d$ and $t)$ the picture is very similar. As one can see the ground state energies of both two- and three-particle systems increase as the screening parameter $\lambda$ is increased, meaning that stability of the systems is weakened by the plasma influence. So that eventually, for large screening, the systems become unbound. By scaling the result of Gomez, Chacham and Mohallem [34] to the actual masses of our systems, we find that the critical 
values of $\lambda, \lambda_{c}$, at which the two-body systems become unbound are about 221, 233 and 237, respectively for $p \mu, d \mu$ and $t \mu$.

By taking the difference between the two-body and the corresponding threebody energies at each value of $\lambda$ we find the proton affinity of $p \mu$, deuteron affinity of $d \mu$ and triton affinity of $t \mu$. These hadronic affinities are plotted in Fig. 3, and given in Tables 1-3. They decrease with $\lambda$ increasing, indicating that the binding of the second proton (deuteron or triton) also weakens with the plasma density increasing. However, this tendency is much weaker than for the first proton (deuteron or triton). The extreme manifestation of such a behavior is that for large values of the screening parameter the proton affinity of the $p \mu$ system is bigger than the binding energy of $p \mu$ itself. The same happens for $d \mu$ and $t \mu$.

Apparently the effect described above is the Thomas collapse [35]: the binding energy per particle is larger for a three-body system than for its two-body counterpart. Such an effect can result in binding of the three-body system even if the two-body subsystems are not bound, i.e. for $\lambda \geq \lambda_{c}$. Checking whether such a possibility can be physically realized for $p p \mu, d d \mu$ and $t t \mu$ systems would be of great interest and importance. However this is not the aim of present investigation. The quality of our trial function is good for $\lambda<\lambda_{c}$ but not good enough to represent properly the $\lambda \geq \lambda_{c}$ region.

We would like to understand where such a strong three-body binding comes from in terms of our computation. For this purpose we computed the dipole polarizabilities of the two-particle systems, using the standard linear response theory with a perturbed function chosen as linear combination of eight STO's [12]. The quality of results is again checked against the standard second order perturbation theory results for the plasma-free hydrogen-like systems (see Tables $1-3)$. As the Debye screening increases the polarizability increases very rapidly, reaching extremely large values at large $\lambda$. This indicates that the strong binding of three-particle systems is via polarization effects. This is confirmed by three-particle computations: when computed by including only radial correlations (36-linear-parameter computation; all functions $\eta\left(r_{12}\right)$ in Eq. 3 set equal to 1) the total three-body energy is very poor (see Tables 1-3 and Fig. 3). For large screening the radial correlation is not enough to bind the system. It is the angular correlation which describes the polarization effects properly so that the system turns out to be bound. The effect of the angular correlation on the ground state energy is presented in Fig. 4. For all the systems under consideration the absolute value of the angular correlation contribution decreases when the screening is increased, Fig. 4a. However, this is just a manifestation of the fact that all the Coulomb interaction effects are weakened by the screening. In fact the role of angular correlation relatively increases with $\lambda$, which can be seen from Fig. $4 b$. 


\section{Conclusion and synopsis}

In summary, we have considered the effect of plasma as envisaged by the Debye model on the energy of exotic hadronic systems $p p \mu, d d \mu$ and $t t \mu$. We have explicitly demonstrated the importance of the inclusion of particle correlations in evaluating connected properties. For strong screening the hadronic affinity is larger than the binding energy of the related two-particle system. We have found that the effect is due to an extremely strong polarization of two-particle systems, increasing when the screening is increased. Fact that the binding of three-particle system is stronger the binding of two-particle subsystems is known as the Thomas collapse [35]. In the case of short-range attractive twobody interactions it can lead to the binding of a three-body system even if the two-body subsystems are not bound [35]. We believe this can be the case for the systems considered in this paper and this shall be a subject of our further investigation.

\section{Acknowledgement}

The financial assistance from the Department of Science and Technology (DST), Govt. of India under research grant no. INT/POL/P-4/2004 and SR/S2/LOP-05/2005 are gratefully acknowledged.

\section{References}

[1] Lopez X, Sarasola C, Ugalde JM. J Phys Chem A 1997;101:1804.

[2] Okutsu H, Sako T, Yamanouchi K and Diercksen GHF. J Phys B 2005;38:917.

[3] Winkler P. Phys Rev E 1996;53:5517.

[4] Dai ST, Solovyova A, Winkler P. Phys Rev E 2001;64:016408.

[5] Kar S, Ho YK. Int J Quantum Chem 2006;106:814, ibid 2007;107:353.

[6] Kar S, Ho YK. Chem Phys Lett 2006;424:403.

[7] Saha B, Mukherjee TK, Mukherjee PK, Diercksen GHF. Theo Chem Acc 2002;108:305.

[8] Mukherjee PK, Karwowski J, Diercksen GHF. Chem Phys Lett 2002;363:323.

[9] Saha B, Mukherjee PK, Diercksen GHF. Astron Astrophys 2002;396:337.

[10] Saha B, Mukherjee PK, Bielinska-Waz D, Karwowski J. J Quant Spectrosc Radiat Transfer 2005;92:1, ibid 2003;78:131. 
[11] Sil AN, Saha B, Mukherjee PK. Int J Quantum Chem 2005;104:903.

[12] Sil AN, Mukherjee PK. Int J Quantum Chem 2006;106:465.

[13] Griem HR. Principles and Plasma Spectroscopy. Cambridge: Cambridge University Press; 2005.

[14] Fujimoto T. Plasma Spectroscopy. Oxford: Clarendon Press; 2004.

[15] Ichimaru S. Plasma Physics. California: The Benjamin/Cummings Publishing Company Inc.; 1986.

[16] Gupta U, Rajagopal AK. Phys Rept 1982;87:259.

[17] Akhiezer AI, Akhiezer IA, Polovin RA, Sitenko AG, Stepanov KN. Plasma Electrodynamics, vol. 1: linear response theory. Oxford: Pergamon Press; 1975.

[18] Ichimaru S. Rev Mod Phys 1982;54:1017.

[19] Froelich P. Adv Phys 1992;41:405.

[20] Cohen JC. Review of Fundamental Processes and Applications of Atoms and Ions. Singapore: World Scientific; 1993. p. 61.

[21] Cohen-Tannoudji C, Diu B and Laloë F. Quantum Mechanics, vol 1. New York: John Wiley; 2005. p. 811 and references therein.

[22] Primorac M and Kavacevic K. Phys Rev A 1992;46:5420.

[23] Mc Kenna P and Webster B. J Chem Soc Faraday Trans 1 1985;81:225.

[24] Schweppe J, Gruppe A, Bethge K et al. Phys Rev Lett 1983;51:2261, ibid 1985;54:1761.

[25] Kulpa J and Wycech S. Acta Phys Polon B 1996;27:941.

[26] Bailey DH. ACM Trans Math Softw 1995;21:379 and references therein.

[27] Frolov AM. Phys Rev A 1999;59:4270.

[28] Frolov AM. Phys Rev A 2004;69:022505, ibid 1998;58:4479, J Phys B: At Mol Opt Phys 2002;35:L331, Phys Rev E 2000;62:8740.

[29] Eskandhari MR and Madhavi M. Phys Rev A 2003;68:032511.

[30] Sil AN, Mukherjee TK, Mukherjee PK and Vasu P. Int J Quantum Chem 2007;107:946.

[31] Bhattacharyya S, Sil AN, Mukherjee TK and Mukherjee PK. Phys Plasmas 2007 ; in press.

[32] Saha B, Mukherjee TK and Mukherjee PK. Chem Phys Lett 2003;373:218.

[33] Mukherjee PK, Fritzsche S and Fricke B. Phys Lett A 2006;360:287.

[34] Gomez OA, Chacham H and Mohallem J. Phys Rev A 1994;50:228.

[35] Goy J, Richard J-M and Fleck S. Phys Rev A 1995;52:3511. 


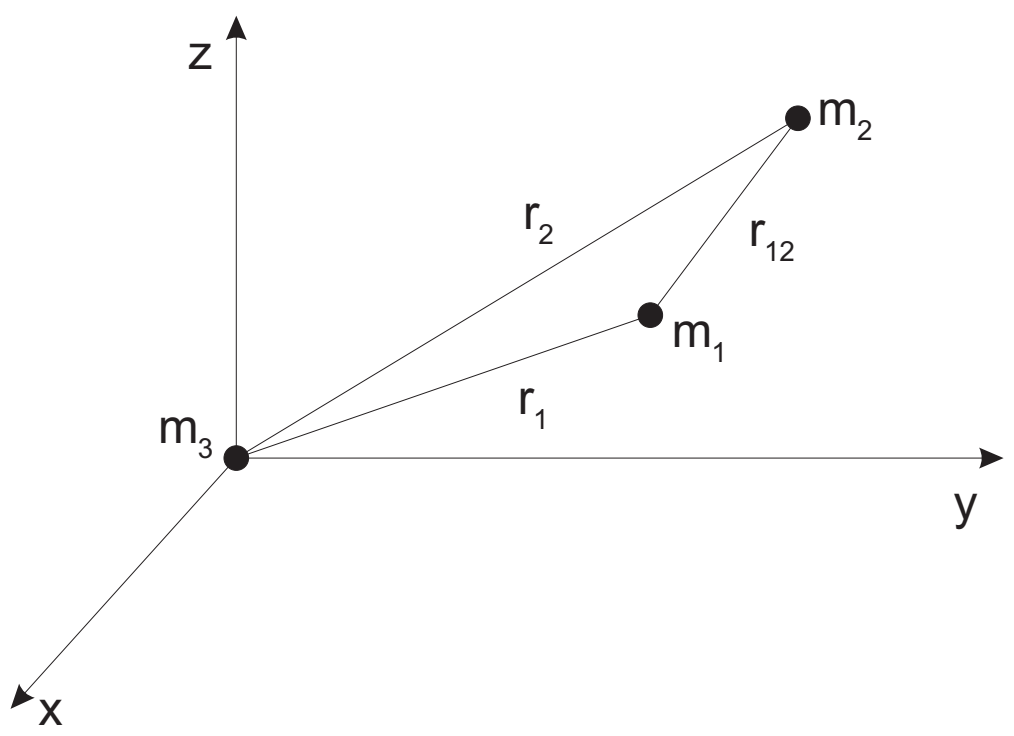

Fig. 1. Coordinates for a three-body system.

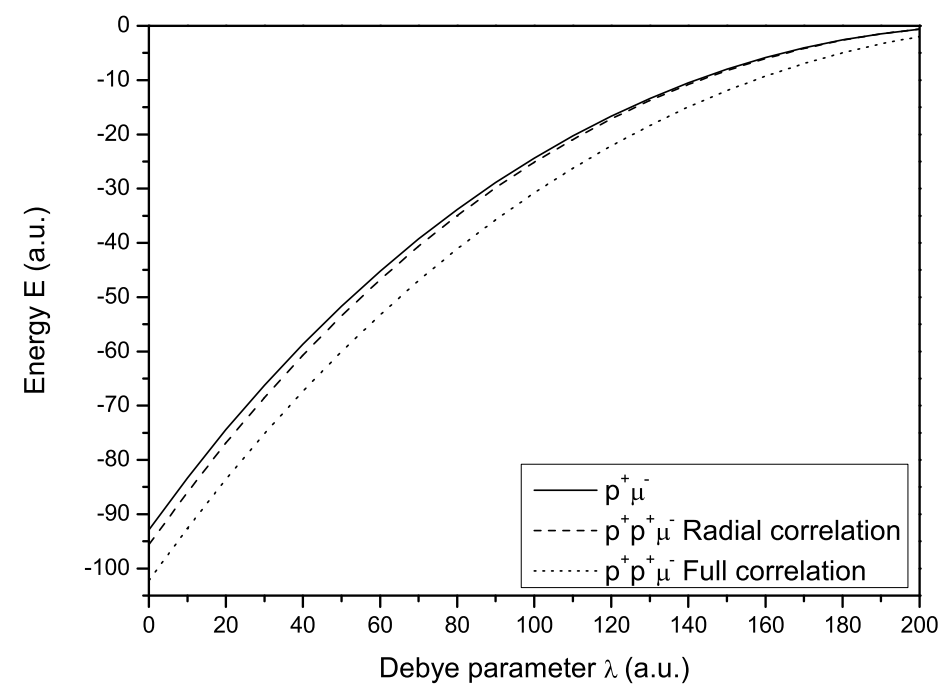

Fig. 2. Energy of $p \mu$ and $p p \mu$ against Debye parameter. 


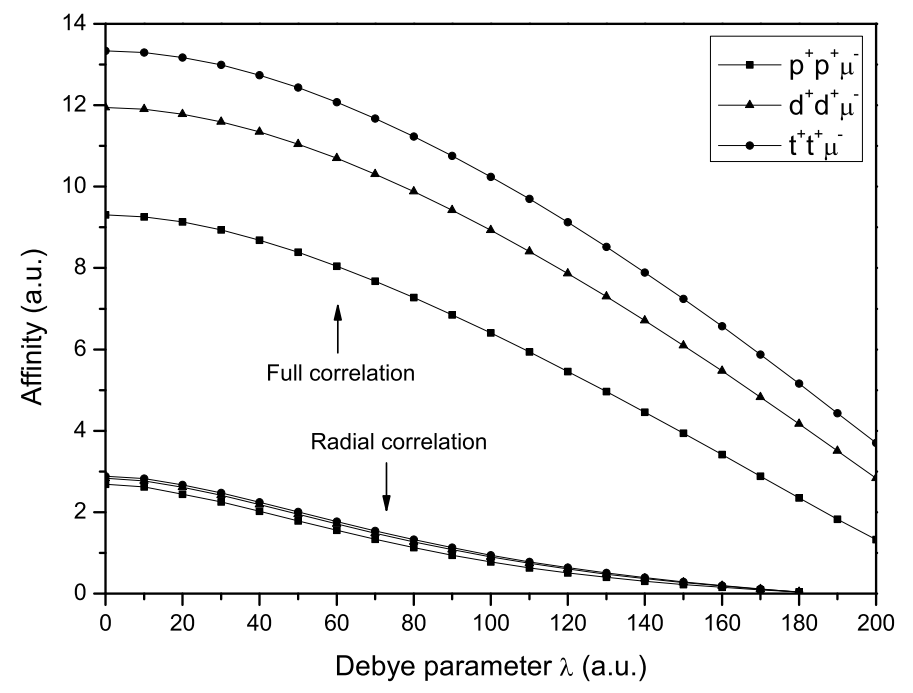

Fig. 3. Hadronic affinity against Debye screening parameter. 

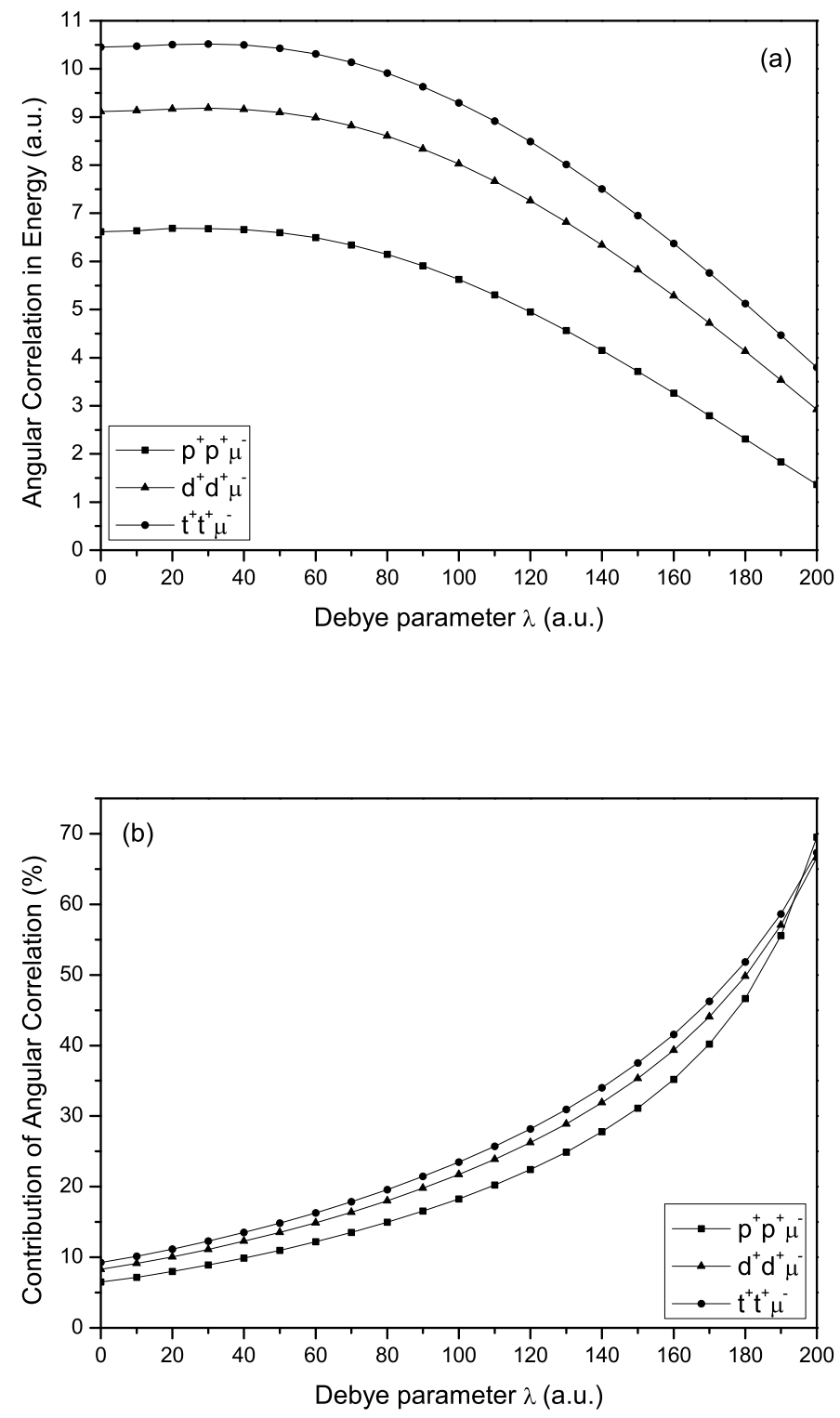

Fig. 4. Contribution of the angular correlation effects to the ground state energy against the Debye screening parameter: a) absolute energy contribution in a.u., b) percentage contribution with respect to the total energy. 
Table 1

Structural properties of $p p \mu$ in Debye plasma.

\begin{tabular}{|c|c|c|c|c|c|c|}
\hline \multirow{3}{*}{$\begin{array}{c}\text { Debye } \\
\text { parameter }^{a} \\
\lambda \text { (a.u.) }\end{array}$} & \multirow{3}{*}{$\begin{array}{c}\text { Plasma } \\
\text { density }^{a} \\
\text { n/c.c. }\end{array}$} & \multirow{3}{*}{$\begin{array}{c}-\mathrm{E}\left(p^{ \pm} \mu^{\mp}\right) \\
\text { (a.u.) }\end{array}$} & \multirow{3}{*}{$\begin{array}{c}\text { Dipole } \\
\text { polarizability } \\
\alpha * 10^{6} \text { (a.u.) }\end{array}$} & \multicolumn{2}{|c|}{$-\mathrm{E}\left(p^{+} p^{+} \mu^{-}\right)$(a.u.) } & \multirow{3}{*}{$\begin{array}{l}\text { Affinity } \\
\text { (a.u.) }\end{array}$} \\
\hline & & & & Radially & Fully & \\
\hline & & & & correlated & correlated & \\
\hline \multirow[t]{2}{*}{0.0} & & 92.9204 & 0.701 & 95.6048 & 102.2235 & 9.3031 \\
\hline & & $92.9204^{b}$ & $0.701^{b}$ & & & \\
\hline 10.0 & $9.87(+26)$ & 83.3105 & 0.710 & 85.9294 & 92.5682 & 9.2577 \\
\hline 20.0 & $3.95(+27)$ & 74.4332 & 0.737 & 76.8751 & 83.5641 & 9.1309 \\
\hline 30.0 & $8.88(+27)$ & 66.2287 & 0.780 & 68.4834 & 75.1649 & 8.9362 \\
\hline 40.0 & $1.58(+28)$ & 58.6487 & 0.841 & 60.6724 & 67.3333 & 8.6846 \\
\hline 50.0 & $2.47(+28)$ & 51.6528 & 0.924 & 53.4389 & 60.0386 & 8.3858 \\
\hline 60.0 & $3.55(+28)$ & 45.2067 & 1.034 & 46.7601 & 53.2540 & 8.0473 \\
\hline 70.0 & $4.84(+28)$ & 39.2808 & 1.180 & 40.6136 & 46.9562 & 7.6754 \\
\hline 80.0 & $6.32(+28)$ & 33.8491 & 1.376 & 34.9780 & 41.1240 & 7.2749 \\
\hline 90.0 & $7.99(+28)$ & 28.8886 & 1.642 & 29.8327 & 35.7385 & 6.8499 \\
\hline 100.0 & $9.87(+28)$ & 24.3787 & 2.012 & 25.1580 & 30.7825 & 6.4038 \\
\hline 110.0 & $1.19(+29)$ & 20.3009 & 2.541 & 20.9352 & 26.2403 & 5.9394 \\
\hline 120.0 & $1.42(+29)$ & 16.6384 & 3.326 & 17.1465 & 22.0973 & 5.4589 \\
\hline 130.0 & $1.67(+29)$ & 13.3759 & 4.540 & 13.7749 & 18.3403 & 4.9644 \\
\hline 140.0 & $1.93(+29)$ & 10.4993 & 6.523 & 10.8046 & 14.9573 & 4.4579 \\
\hline 150.0 & $2.22(+29)$ & 7.9957 & 9.996 & 8.2203 & 11.9369 & 3.9412 \\
\hline 160.0 & $2.52(+29)$ & 5.8529 & 16.630 & 6.0077 & 9.2691 & 3.4162 \\
\hline 170.0 & $2.85(+29)$ & 4.0596 & 30.600 & 4.1533 & 6.9447 & 2.8851 \\
\hline 180.0 & $3.19(+29)$ & 2.6044 & 62.370 & 2.6435 & 4.9559 & 2.3515 \\
\hline 190.0 & $3.56(+29)$ & 1.4722 & 136.100 & 1.4643 & 3.2960 & 1.8238 \\
\hline 200.0 & $3.94(+29)$ & 0.6365 & 299.200 & 0.5974 & 1.9603 & 1.3238 \\
\hline 210.0 & $4.35(+29)$ & 0.0508 & 651.900 & & 0.9471 & 0.8963 \\
\hline
\end{tabular}

${ }^{a}$ We have chosen here a typical case of $1000 \mathrm{eV}$ plasma. The plasma screening parameter $\lambda$ chosen here gives the value of the possible electron density from Eq. (2). ${ }^{b}$ Standard second order perturbation theory result for the hydrogen-like system. 
Table 2

Structural properties of $d d \mu$ in Debye plasma.

\begin{tabular}{|c|c|c|c|c|c|c|}
\hline \multirow{3}{*}{$\begin{array}{c}\text { Debye } \\
\text { parameter }^{a} \\
\lambda(\text { a.u. })\end{array}$} & \multirow{3}{*}{$\begin{array}{c}\text { Plasma } \\
\text { density }^{a} \\
\text { n/c.c. }\end{array}$} & \multirow{3}{*}{$\begin{array}{c}-\mathrm{E}\left(d^{ \pm} \mu^{\mp}\right) \\
\text { (a.u.) }\end{array}$} & \multirow{3}{*}{$\begin{array}{c}\text { Dipole } \\
\text { polarizability } \\
\alpha * 10^{6} \text { (a.u.) }\end{array}$} & \multicolumn{2}{|c|}{$-\mathrm{E}\left(d^{+} d^{+} \mu^{-}\right)$(a.u.) } & \multirow{3}{*}{$\begin{array}{l}\text { Affinity } \\
\text { (a.u.) }\end{array}$} \\
\hline & & & & Radially & Fully & \\
\hline & & & & correlated & correlated & \\
\hline \multirow[t]{2}{*}{0.0} & & 97.8708 & 0.600 & 100.7020 & 109.8165 & 11.9457 \\
\hline & & $97.8708^{b}$ & $0.600^{b}$ & & & \\
\hline 10.0 & $9.87(+26)$ & 88.2417 & 0.607 & 91.0103 & 100.1456 & 11.9038 \\
\hline 20.0 & $3.95(+27)$ & 79.3114 & 0.627 & 81.9278 & 91.0954 & 11.7840 \\
\hline 30.0 & $8.88(+27)$ & 71.0249 & 0.660 & 73.4384 & 82.6204 & 11.5955 \\
\hline 40.0 & $1.58(+28)$ & 63.3377 & 0.708 & 65.5224 & 74.6842 & 11.3465 \\
\hline 50.0 & $2.47(+28)$ & 56.2122 & 0.771 & 58.1591 & 67.2567 & 11.0445 \\
\hline 60.0 & $3.55(+28)$ & 49.6164 & 0.853 & 51.3277 & 60.3122 & 10.6958 \\
\hline 70.0 & $4.84(+28)$ & 43.5227 & 0.961 & 45.0079 & 53.8281 & 10.3054 \\
\hline 80.0 & $6.32(+28)$ & 37.9064 & 1.103 & 39.1799 & 47.7844 & 9.8780 \\
\hline 90.0 & $7.99(+28)$ & 32.7461 & 1.292 & 33.8247 & 42.1632 & 9.4171 \\
\hline 100.0 & $9.87(+28)$ & 28.0222 & 1.548 & 28.9242 & 36.9481 & 8.9259 \\
\hline 110.0 & $1.19(+29)$ & 23.7172 & 1.901 & 24.4609 & 32.1243 & 8.4071 \\
\hline 120.0 & $1.42(+29)$ & 19.8152 & 2.405 & 20.4181 & 27.6781 & 7.8629 \\
\hline 130.0 & $1.67(+29)$ & 16.3016 & 3.148 & 16.7802 & 23.5971 & 7.2955 \\
\hline 140.0 & $1.93(+29)$ & 13.1630 & 4.290 & 13.5319 & 19.8695 & 6.7065 \\
\hline 150.0 & $2.22(+29)$ & 10.3871 & 6.141 & 10.6593 & 16.4848 & 6.0977 \\
\hline 160.0 & $2.52(+29)$ & 7.9624 & 9.344 & 8.1486 & 13.4331 & 5.4707 \\
\hline 170.0 & $2.85(+29)$ & 5.8781 & 15.310 & 5.9870 & 10.7054 & 4.8273 \\
\hline 180.0 & $3.19(+29)$ & 4.1238 & 27.250 & 4.1618 & 8.2936 & 4.1698 \\
\hline 190.0 & $3.56(+29)$ & 2.6883 & 52.290 & 2.6597 & 6.1904 & 3.5021 \\
\hline 200.0 & $3.94(+29)$ & 1.5558 & 104.700 & 1.4656 & 4.3895 & 2.8337 \\
\hline 210.0 & $4.35(+29)$ & 0.6999 & 209.600 & 0.5585 & 2.8861 & 2.1862 \\
\hline 220.0 & $4.77(+29)$ & 0.0786 & 415.200 & & 1.6768 & 1.5982 \\
\hline
\end{tabular}

${ }^{\bar{a}}$ We have chosen here a typical case of $1000 \mathrm{eV}$ plasma. The plasma screening parameter $\lambda$ chosen here gives the value of the possible electron density from Eq. (2). ${ }^{b}$ Standard second order perturbation theory result for the hydrogen-like system. 
Table 3

Structural properties of $t t \mu$ in Debye plasma.

\begin{tabular}{|c|c|c|c|c|c|c|}
\hline \multirow{3}{*}{$\begin{array}{c}\text { Debye } \\
\text { parameter }^{a} \\
\lambda \text { (a.u.) }\end{array}$} & \multirow{3}{*}{$\begin{array}{c}\text { Plasma } \\
\text { density }^{a} \\
\text { n/c.c. }\end{array}$} & \multirow{3}{*}{$\begin{array}{c}-\mathrm{E}\left(t^{ \pm} \mu^{\mp}\right) \\
\text { (a.u. })\end{array}$} & \multirow{3}{*}{$\begin{array}{c}\text { Dipole } \\
\text { polarizability } \\
\alpha * 10^{6} \text { (a.u.) }\end{array}$} & \multicolumn{2}{|c|}{$-\mathrm{E}\left(t^{+} t^{+} \mu^{-}\right)$(a.u.) } & \multirow{3}{*}{$\begin{array}{l}\text { Affinity } \\
\text { (a.u.) }\end{array}$} \\
\hline & & & & Radially & Fully & \\
\hline & & & & correlated & correlated & \\
\hline \multirow[t]{2}{*}{0.0} & & 99.6363 & 0.569 & 102.5203 & 112.9718 & 13.3355 \\
\hline & & $99.6363^{b}$ & $0.569^{b}$ & & & \\
\hline 10.0 & $9.87(+26)$ & 90.0009 & 0.575 & 92.8231 & 103.2953 & 13.2944 \\
\hline 20.0 & $3.95(+27)$ & 81.0528 & 0.594 & 83.7245 & 94.2289 & 13.1761 \\
\hline 30.0 & $8.88(+27)$ & 72.7389 & 0.625 & 75.2091 & 85.7272 & 12.9883 \\
\hline 40.0 & $1.58(+28)$ & 65.0157 & 0.667 & 67.2580 & 77.7540 & 12.7383 \\
\hline 50.0 & $2.47(+28)$ & 57.8467 & 0.728 & 59.8511 & 70.2793 & 12.4326 \\
\hline 60.0 & $3.55(+28)$ & 51.2006 & 0.780 & 52.9684 & 63.2776 & 12.0770 \\
\hline 70.0 & $4.84(+28)$ & 45.0504 & 0.897 & 46.5902 & 56.7266 & 11.6762 \\
\hline 80.0 & $6.32(+28)$ & 39.3721 & 1.024 & 40.6972 & 50.6066 & 11.2345 \\
\hline 90.0 & $7.99(+28)$ & 34.1444 & 1.192 & 35.2710 & 44.8999 & 10.7555 \\
\hline 100.0 & $9.87(+28)$ & 29.3484 & 1.418 & 30.2939 & 39.5905 & 10.2421 \\
\hline 110.0 & $1.19(+29)$ & 24.9668 & 1.727 & 25.7489 & 34.6639 & 9.6971 \\
\hline 120.0 & $1.42(+29)$ & 20.9839 & 2.161 & 21.6198 & 30.1065 & 9.1226 \\
\hline 130.0 & $1.67(+29)$ & 17.3855 & 2.792 & 17.8911 & 25.9061 & 8.5206 \\
\hline 140.0 & $1.93(+29)$ & 14.1583 & 3.744 & 14.5483 & 22.0512 & 7.8928 \\
\hline 150.0 & $2.22(+29)$ & 11.2904 & 5.251 & 11.5773 & 18.5315 & 7.2410 \\
\hline 160.0 & $2.52(+29)$ & 8.7702 & 7.766 & 8.9649 & 15.3370 & 6.5668 \\
\hline 170.0 & $2.85(+29)$ & 6.5870 & 12.210 & 6.6984 & 12.4590 & 5.8720 \\
\hline 180.0 & $3.19(+29)$ & 4.7303 & 20.460 & 4.7653 & 9.8892 & 5.1589 \\
\hline 190.0 & $3.56(+29)$ & 3.1881 & 36.210 & 3.1527 & 7.6201 & 4.4320 \\
\hline 200.0 & $3.94(+29)$ & 1.9442 & 66.080 & 1.8459 & 5.6452 & 3.7010 \\
\hline 210.0 & $4.35(+29)$ & 0.9737 & 120.600 & 0.8258 & 3.9589 & 2.9852 \\
\hline 220.0 & $4.77(+29)$ & 0.2401 & 217.400 & 0.0638 & 2.5569 & 2.3168 \\
\hline
\end{tabular}

$\overline{\bar{a}}$ We have chosen here a typical case of $1000 \mathrm{eV}$ plasma. The plasma screening parameter $\lambda$ chosen here gives the value of the possible electron density from Eq. (2). ${ }^{b}$ Standard second order perturbation theory result for the hydrogen-like system. 